\title{
Character Large Object
}

National Cancer Institute

\section{Source}

National Cancer Institute. Character Large Object. NCI Thesaurus. Code C48291.

A data type for holding large chunks of text. 